\title{
Change in the Recurrence Pattern and Predictors over Time after Complete Cure of Hepatocellular Carcinoma
}

\author{
Han Ah Lee1, Young-Sun Lee', Beom Kyung Kim², Young Kul Jung' ${ }^{1}$, Seung Up Kim², Jun Yong Park², Ji Hoon \\ $\mathrm{Kim}^{1}$, Hyunggin $\mathrm{An}^{3}$, Do Young Kim², Hyung Joon Yim, Sang Hoon Ahn², Jong Eun Yeon', Kwan Soo Byun', \\ Kwang-Hyub Han², Soon Ho Um ${ }^{1}$, and Yeon Seok Seo ${ }^{1}$ \\ ${ }^{1}$ Department of Internal Medicine, Korea University College of Medicine, ${ }^{2}$ Department of Internal Medicine, Yonsei University College \\ of Medicine, and ${ }^{3}$ Department of Biostatistics, Korea University College of Medicine, Seoul, Korea
}

Article Info
Received March 16, 2020
Revised April 24, 2020
Accepted April 28, 2020
Published online August 26, 2020
Corresponding Author
Yeon Seok Seo
ORCID https://orcid.org/0000-0003-4171-6331
E-mail drseo@korea.ac.kr
Seung Up Kim
ORCID https://orcid.org/0000-0002-9658-8050
E-mail KSUKOREA@yuhs.ac

E-mail KSUKOREA@yuhs.ac

\begin{abstract}
Background/Aims: We investigated changes in recurrence rates and significant recurrence predictors over time after complete cure of hepatocellular carcinoma (HCC).

Methods: A total of 1,491 patients with first-time diagnosis of Barcelona Clinic Liver Cancer stage A HCC, completely cured by treatment between 2007 and 2016, were recruited from two Korean tertiary institutes.

Results: The mean age of the population (1,144 men and 347 women) was 58.6 years. Of the total population, 914 patients $(61.3 \%)$ had liver cirrhosis. Nine-hundred and forty-one $(63.1 \%)$ and $550(36.9 \%)$ patients were treated with surgical resection and radiofrequency ablation (RFA), respectively. One-year cumulative incidences of HCC recurrence were $14.3 \%, 9.9 \%$, and $5.1 \%$ from the time of treatment, 3 years after treatment, and 5 years after treatment, respectively. Upon multivariate analysis, multiple tumors, maximal tumor size $\geq 3 \mathrm{~cm}$, and high Model for EndStage Liver Disease scores were independently associated with increased HCC recurrence risk from the time of treatment and 1 and 2 years after curative treatment (all $p<0.05$, except for maximal tumor size $\geq 3 \mathrm{~cm}$ for recurrence 2 years after treatment). Meanwhile, liver cirrhosis and RFA were independently associated with the increased $\mathrm{HCC}$ recurrence risk for almost all time points (liver cirrhosis: all $p<0.05$; RFA: all $p<0.005$ except for recurrence from 5 years after treatment).

Conclusions: The recurrence rate of $\mathrm{HCC}$ after curative treatment gradually decreased over time. Two years after treatment, when tumor-related factors lose their prognostic implications, may be used as a cutoff to define the boundary between early and late recurrence of HCC. (Gut Liver 2021;15:420-429)
\end{abstract}

Key Words: Recurrence; Risk factor; Barcelona Clinic Liver Cancer stage A; Carcinoma, hepatocellular

\section{INTRODUCTION}

Hepatocellular carcinoma (HCC) is the fifth most common tumor and second most common cause of cancerrelated death globally. ${ }^{1}$ In patients with Barcelona Clinic Liver Cancer (BCLC) stage A HCC, surgical resection or radiofrequency ablation (RFA) are usually recommended. ${ }^{2-4}$ Though median survival reaches $50 \%$ to $70 \%$ at 5 years after curative treatment, recurrence rate is still high. ${ }^{5-7}$ Because recurrence is the main cause of mortality in HCC patients, ${ }^{8,9}$ identification of recurrence patterns and predictive factors is crucial.

Previous studies have suggested two distinct types of HCC recurrence: tumors originating from subclinical metastasis of primary tumors and multicentric second primary tumor arising from diseased liver, ${ }^{10,11}$ so called "early" and "late" recurrence, respectively. ${ }^{12}$ Because each recurrence is based on different biology, this difference has to be accounted for establishing surveillance and treatment strategy for HCC recurrence. ${ }^{13}$ 
However, the lack of reliable markers applicable in clinical setting makes it difficult to differentiate these recurrence types. Early recurrence is thought to be associated with tumor-related factors, and late recurrence with the liver disease-related factors. Previous studies assessed the prognostic implications of various risk factors with respect to early and late HCC recurrence. However, those studies have shown conflicting results. ${ }^{12,14-18}$ These disparities might be due to heterogeneous study population having various clinicopathological characteristics. In addition, the change of recurrence rates after curative treatment has also not been well assessed in previous studies, possibly arousing confusion in establishing surveillance strategy for HCC recurrence. ${ }^{19}$

To counteract these shortcomings, we conducted this large, multicenter retrospective cohort study in patients with first-time diagnosis of BCLC A HCC who were completely cured with surgical resection or RFA. We investigated changes in recurrence rates and independent predictors of HCC recurrence over time after curative treatment.

\section{MATERIALS AND METHODS}

\section{Study design and subjects}

We retrospectively reviewed medical records of 4,487 patients with first-time diagnosis of HCC at Korea University Anam Hospital and Yonsei University Severance Hospital between 2007 and 2016. In total, 1,491 patients with BCLC stage A HCC, who were completely cured with surgical resection or RFA were considered eligible. Exclusion criteria were: (1) previous history of treatment for HCC; (2) not treated or treated with methods other than surgical resection or RFA; (3) Child-Pugh class B and C; (4) Eastern Cooperative Oncology Group performance status $>2$; (5) presence of extrahepatic metastasis; and (6) combined malignancy other than HCC.

Flowsheet of all included patients is presented in Fig. 1. Patients with chronic hepatitis B virus (HBV) or hepatitis $\mathrm{C}$ virus (HCV) infection were treated for viral hepatitis as per treatment guidelines. ${ }^{20-23}$

The study protocol was in accordance with the Declaration of Helsinki guidelines. The need for written informed consent was waived because of the retrospective nature of this study. The study procedure was approved by the Institutional Review Boards of Korea University Anam Hos-

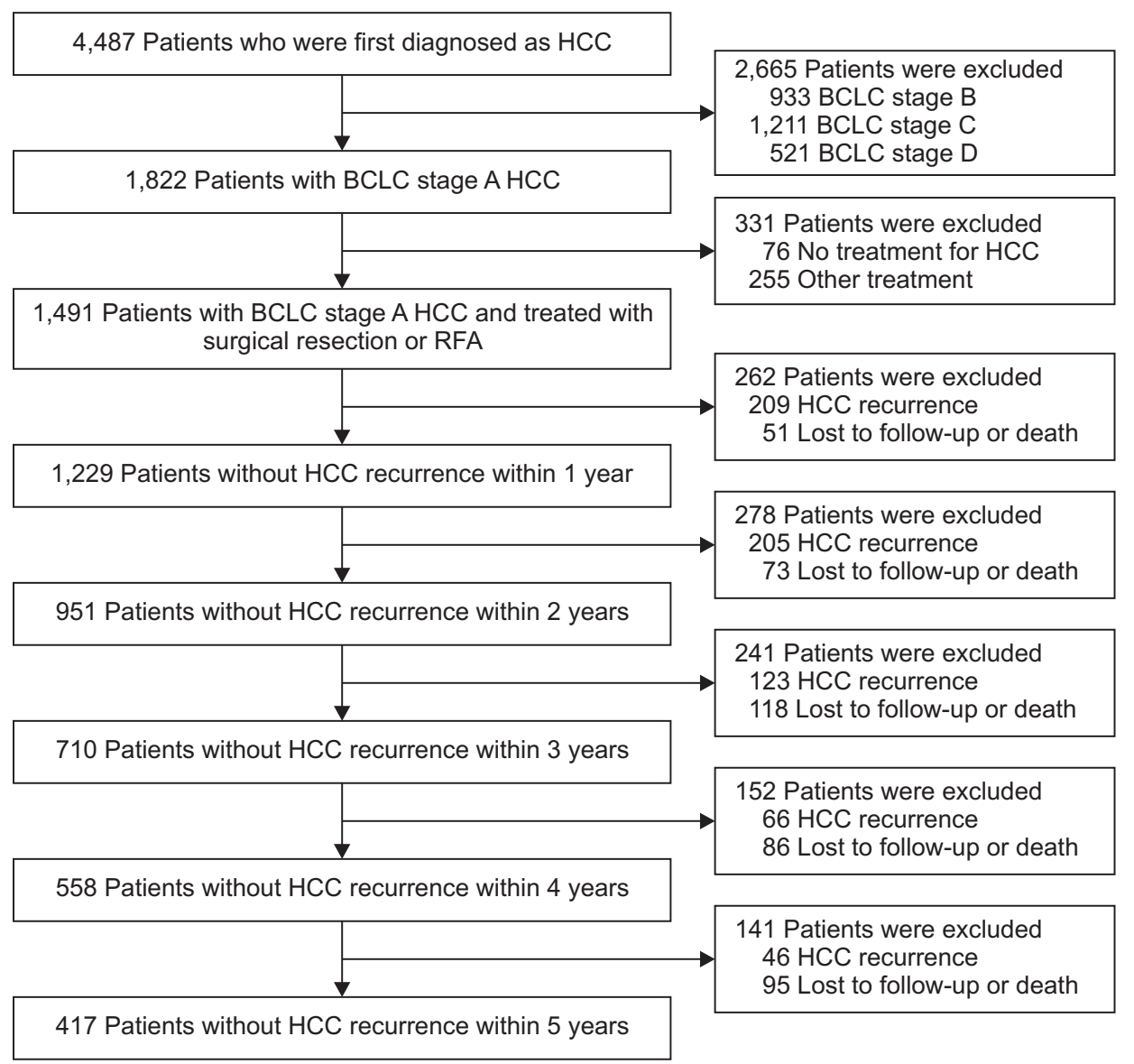

Fig. 1. Flowchart of the patient population.

HCC, hepatocellular carcinoma; BCLC, Barcelona Clinic Liver Cancer; RFA, radiofrequency ablation. 
pital and Yonsei University Health System (IRB number: 2016AN0362).

\section{Diagnosis and staging}

Diagnosis of HCC was based on noninvasive criteria and/or pathology in cirrhotic patients, and pathology in non-cirrhotic patients. Noninvasive criteria were based on identification of typical hallmarks of HCC, obtained by multiphasic computed tomography (CT) or multiphase magnetic resonance imaging. ${ }^{24}$ BCLC staging system was used for staging HCC. ${ }^{25}$

\section{Curative treatment and evaluation of treatment response}

Type of treatment (surgical resection vs RFA) was determined according to the clinician's decision based on the clinical practice guideline for HCC in Korea. ${ }^{2,26}$

Treatment response after curative treatment was evaluated at 1 month after treatment. Complete response was defined as disappearance of any intratumoral arterial enhancement in all target lesions, as evaluated by multiphasic CT or magnetic resonance imaging at 1 month after treatment. ${ }^{27}$

\section{Recurrence surveillance after curative treatment}

After curative treatment, patients were surveilled periodically at outpatient clinics. Surveillance for recurrence consisted of imaging techniques by multiphasic CT or magnetic resonance imaging and blood tests including known predictors of HCC recurrence. Due to a lack of evidence-based guidelines for recurrence surveillance, screening was conducted every 1 to 6 months as determined by the clinician's decision.

Intrahepatic HCC recurrence was defined by the same diagnostic criteria applied for initial diagnosis. Extrahepatic recurrence was evaluated by $\mathrm{CT}$ or bone scan performed at the discretion of the clinician.

\section{Data collection and primary endpoint assessment}

We investigated patients' age, gender, number of tumors, maximal tumor size, international normalized ratio, serum bilirubin, creatinine, alpha-fetoprotein (AFP), and protein induced by vitamin $\mathrm{K}$ absence or antagonist II (PIVKA-II) levels at the time of HCC diagnosis. The etiology of liver disease were classified as $\mathrm{HBV}, \mathrm{HCV}$, alcoholic liver disease and nonalcoholic fatty liver disease, based on serum hepatitis B surface antigen, hepatitis C antibody, imaging studies, and clinical history.

\section{Outcomes}

Primary outcome was the change in HCC recurrence rate. Secondary outcome was the change of independent predictors for HCC recurrence over time after curative treatment. To analyze the change in recurrence rate and predictors of HCC recurrence, we performed different statistical analyses from previous studies, annually censoring patients who developed recurrence and those lost to follow-up and death at the time of each event. Variables at the time of treatment were analyzed in total patients for rate and independent predictors of HCC recurrence from the time of treatment. Then, these analyses were repeated separately after annual exclusion of censored patients as follows; in remaining patients without censoring at each time points (1,2,3,4, and 5 years after curative treatment), variables measured at each time point were analyzed for rate and independent predictors of HCC recurrence from each time point, respectively (Fig. 1).

\section{Statistical analysis}

Data are expressed as means \pm standard deviation or numbers (\%), as appropriate. The significance of differences among continuous and categorical variables was examined using the Student t-test and chi-square test, respectively. Cumulative incidence of HCC recurrence was evaluated by the Kaplan-Meier method. Multivariate analysis was performed to identify independent predictors of HCC recurrence using the Cox proportional hazards model.

Statistical analyses were conducted using Statistical Analysis Systems version 9.2 (SAS Institute Inc., Cary, NC, USA) and R software V.3.0 (R Foundation for Statistical Computing, Vienna, Austria; http://cran.r-project.org/). Two-sided p-values $<0.05$ were considered indicative of statistical significance.

\section{RESULTS}

\section{Baseline characteristics of the study population}

In total, 1,491 patients who were cured of BCLC stage A HCC through surgical resection or RFA were finally selected for the statistical analysis (Fig. 1). The baseline characteristics of the study population are listed in Table 1 . The mean age was 58.6 years, and men were predominant $(n=1,144$, $76.7 \%)$. In the study population, chronic HBV infection was the most frequent underlying liver disease $(n=1,152$, 77.3\%) followed by chronic HCV infection ( $\mathrm{n}=137,9.2 \%)$. Of the total population, 914 patients (61.3\%) had liver cirrhosis. The mean number of tumors and mean size of maximal tumor were 1.1 and $2.8 \mathrm{~cm}$, respectively. Serum AFP and PIVKA-II levels were $1.3 \pm 0.9 \log _{10} \mathrm{ng} / \mathrm{mL}$ and $1.8 \pm 0.7 \log _{10} \mathrm{mAU} / \mathrm{mL}$, respectively. 
Table 1. Baseline Characteristics of Patients with HCC Who Were Cured of BCLC Stage A HCC with Surgical Resection or RFA

\begin{tabular}{|c|c|c|c|c|}
\hline Characteristics & All patients $(n=1,491)$ & $\begin{array}{l}\text { Patients treated with resection } \\
\qquad(\mathrm{n}=941)\end{array}$ & $\begin{array}{l}\text { Patients treated with RFA } \\
\qquad(n=550)\end{array}$ & $\mathrm{p}$-value \\
\hline Age, yr & $58.6 \pm 10.3$ & $57.4 \pm 9.9$ & $60.7 \pm 10.6$ & $<0.001$ \\
\hline Male sex & $1,144(76.7)$ & $747(79.4)$ & 397 (72.2) & 0.001 \\
\hline Etiology & & & & $<0.001$ \\
\hline HBV & $1,152(77.3)$ & 779 (82.8) & 373 (67.8) & \\
\hline $\mathrm{HCV}$ & 137 (9.2) & $49(5.2)$ & $88(16.0)$ & \\
\hline Alcohol & $77(5.2)$ & $32(3.4)$ & $45(8.2)$ & \\
\hline NAFLD & $99(6.6)$ & $67(7.1)$ & $32(5.8)$ & \\
\hline Other & $26(1.7)$ & 14 (1.5) & $12(2.2)$ & \\
\hline Liver cirrhosis & 914 (61.3) & 478 (50.8) & 436 (79.3) & $<0.001$ \\
\hline MELD score & $7.6 \pm 2.2$ & $7.0 \pm 1.5$ & $8.6 \pm 2.8$ & $<0.001$ \\
\hline No. of tumors & $1.1 \pm 0.4$ & $1.1 \pm 0.2$ & $1.2 \pm 0.5$ & $<0.001$ \\
\hline Size of maximal tumor, $\mathrm{cm}$ & $2.9 \pm 1.8$ & $3.4 \pm 2.0$ & $2.0 \pm 0.9$ & $<0.001$ \\
\hline AFP, $\log _{10} n g / m L$ & $1.3 \pm 0.9$ & $1.4 \pm 1.0$ & $1.2 \pm 0.8$ & $<0.001$ \\
\hline PIVKA-II, $\log _{10} \mathrm{mAU} / \mathrm{mL}$ & $1.8 \pm 0.7$ & $1.9 \pm 0.7$ & $1.7 \pm 0.6$ & $<0.001$ \\
\hline
\end{tabular}

Data are presented as mean \pm SD or number $(\%)$.

HCC, hepatocellular carcinoma; BCLC, Barcelona Clinic Liver Cancer; RFA, radiofrequency ablation; HBV, hepatitis B virus; HCV, hepatitis C virus; NAFLD, nonalcoholic fatty liver disease; MELD, Model for End-Stage Liver Disease; AFP, alpha-fetoprotein; PIVKA-II, protein induced by vitamin K absence or antagonist II.

\section{Comparison between patients treated with surgical resection and RFA}

Among all the patients, 941 (63.1\%) were treated with surgical resection and 550 (36.9\%) with RFA. As compared to patients treated with RFA, those treated with surgical resection had significantly younger age (mean 57.4 years vs 60.7 years, $\mathrm{p}<0.001$ ), lower MELD score (mean 7.0 vs 8.6, $\mathrm{p}<0.001)$, higher proportion of men $(79.4 \%$ vs $72.2 \%$, $\mathrm{p}=0.001)$, higher prevalence of HBV infection $(82.8 \%$ vs $67.8 \%, \mathrm{p}<0.001)$, lower prevalence of HCV infection (5.2\% vs $16.0 \%, p<0.001)$, lower prevalence of alcoholic liver disease ( $3.4 \%$ vs $8.2 \%, \mathrm{p}<0.001)$, lower prevalence of liver cirrhosis ( $50.8 \%$ vs $79.3 \%$, $\mathrm{p}<0.001$ ), lower number of tumors (mean 1.1 vs $1.2, \mathrm{p}<0.001$ ), larger size of maximal tumor (mean $3.4 \mathrm{~cm}$ vs $2.0 \mathrm{~cm}, \mathrm{p}<0.001$ ) and higher level of serum AFP $\left(1.4 \pm 1.0 \log _{10} \mathrm{ng} / \mathrm{mL}\right.$ vs $1.2 \pm 0.8 \log _{10} \mathrm{ng} / \mathrm{mL}$, $\mathrm{p}<0.001)$ and PIVKA-II $\left(1.9 \pm 0.7 \log _{10} \mathrm{mAU} / \mathrm{mL}\right.$ vs $1.7 \pm 0.6$ $\log _{10} \mathrm{mAU} / \mathrm{mL}, \mathrm{p}<0.001$ ) (Table 1).

\section{Changes in the recurrence rate of $\mathrm{HCC}$ during the follow-up}

During the follow-up period (median, 58.6 months), 693 patients (46.5\%) developed HCC recurrence. After excluding censored patients at each time points, the cumulative incidence rates of HCC recurrence in each population without recurrence were calculated from the time of treatment, and from 1, 2, 3, 4, and 5 years after treatment.

The cumulative incidences of HCC recurrence were $0.1 \%, 4.7 \%, 10.0 \%$ and $14.3 \%$ at $3,6,9$, and 12 months after treatment, respectively. In 1,229 patients without HCC recurrence within 1 year of treatment, the cumulative inci- dences of recurrence from 1 year after treatment were $5.2 \%$, $10.5 \%, 14.1 \%$, and $17.1 \%$ at $3,6,9$, and 12 months, respectively. In 710 patients without HCC recurrence within 3 years of treatment, the cumulative incidences of recurrence from 3 years after treatment were $2.7 \%, 5.3 \%, 8.3 \%$, and $9.9 \%$ at $3,6,9$, and 12 months, respectively. In 417 patients without HCC recurrence within 5 years, the cumulative incidences of recurrence from 5 years after treatment were $1.5 \%, 3.1 \%, 5.1 \%$, and $6.8 \%$ at $3,6,9$, and 12 months after 5 years, respectively (Fig. 2).

Although the cumulative incidences of HCC recurrence decreased with time progression in both patients with and without cirrhosis, the incidences were significantly higher in patients with cirrhosis than in those without cirrhosis in all time points $(\mathrm{p}<0.001)$. Similarly, although the cumulative incidences of HCC recurrence decreased with time progression in both patients treated with resection and those treated with RFA, the incidences were significantly higher in patients treated with RFA than in those treated with resection in all time points $(\mathrm{p}<0.001)$.

\section{Changes in the characteristics of the study population with time progression}

After excluding censored patients at each time points, characteristics of each population without HCC recurrence were analyzed at the time of treatment, and at 1, 2, 3, 4 , and 5 years after treatment, respectively. There were no changes in the mean age and gender distribution with time progression. The mean MELD score decreased from time of treatment to 1 year after treatment (7.6 at time of treatment and 7.2 at 1 year after treatment), but there were no 


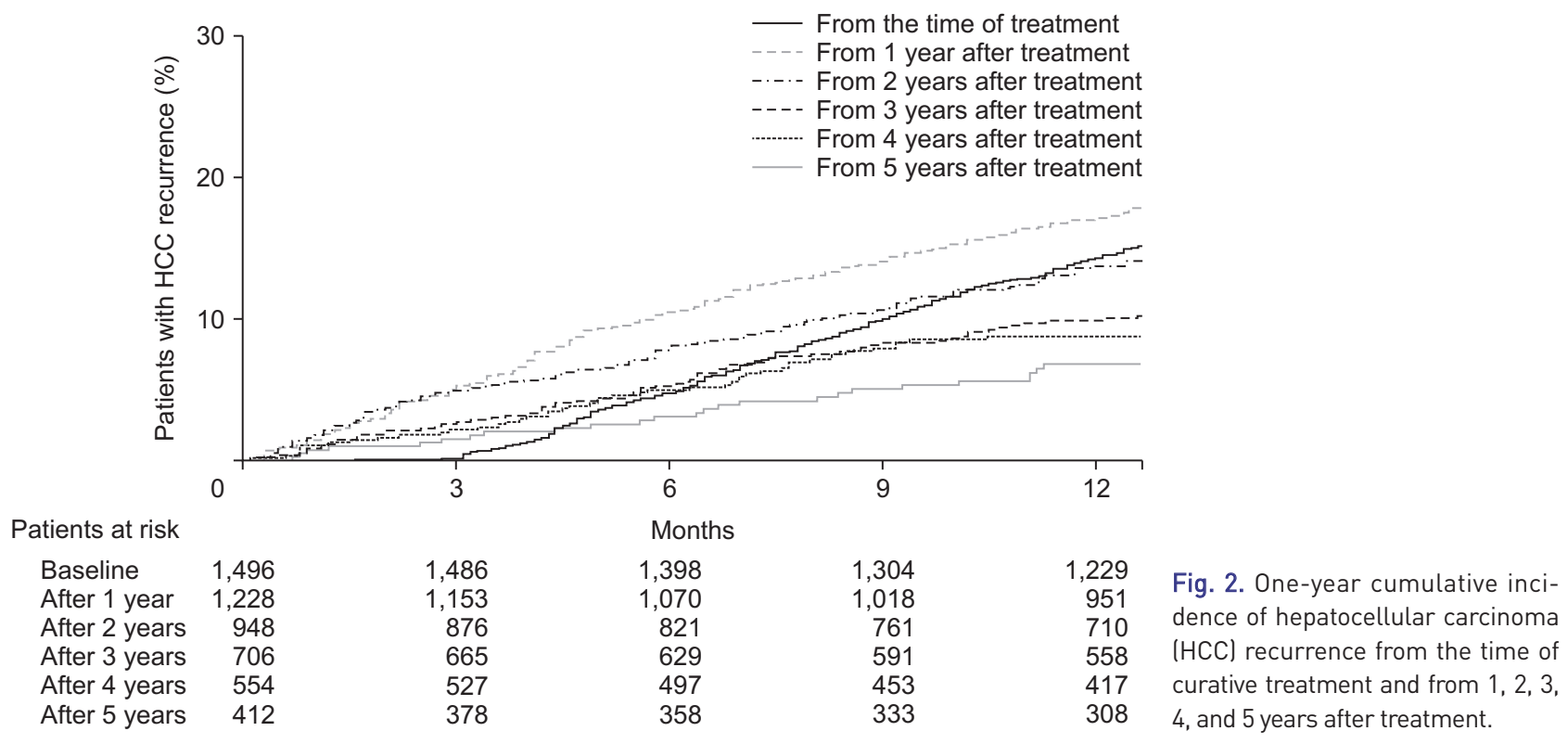

significant changes thereafter. The proportion of patients with chronic HBV infection $(77.3 \%$ at the time of treatment and $85.0 \%$ at 5 years after treatment) and that of patients who with resection $(63.0 \%$ at the time of treatment and $78.6 \%$ at 5 years after treatment) increased with time progression, while the proportion of patients with liver cirrhosis decreased with time progression $(61.4 \%$ at the time of treatment and $48.8 \%$ at 5 years after treatment) (Table 2).

\section{Predictors of HCC recurrence at the time of treatment}

The results of multivariate and univariate Cox-regression analyses for predictors of HCC recurrence at each time point are presented in Table 3 and Supplementary Table 1, respectively. Multivariate analyses revealed that combined liver cirrhosis (hazard ratio [HR], 2.252; 95\% confidence interval [CI], 1.861 to 2.726; $\mathrm{p}<0.001$ ), treated with RFA rather than resection (HR, 1.848; 95\% CI, 1.541 to 2.216; $\mathrm{p}<0.001$ ), multiple tumors (HR, $1.500 ; 95 \% \mathrm{CI}$, 1.254 to 1.793 ; $\mathrm{p}<0.001$ ), maximal tumor size $\geq 3 \mathrm{~cm}$ (HR, 1.112; $95 \%$ CI, 1.060 to 1.166 ; $\mathrm{p}<0.001)$, higher MELD score (HR, 1.038; 95\% CI, 1.003 to $1.074 ; \mathrm{p}=0.031$ ), higher serum AFP (HR, 1.142; 95\% CI, 1.050 to $1.242 ; \mathrm{p}=0.002$ ) and higher PIVKA-II (HR, 1.176; 95\% CI, 1.048 to 1.319; $\mathrm{p}=0.006$ ) levels were significantly associated with increased risk of HCC recurrence from the time of treatment.

\section{Changes in the predictors of $\mathrm{HCC}$ recurrence over time}

After excluding censored patients at each time points, variables collected at the time of treatment were assessed for their prognostic implications with respect to HCC recurrence from 1, 2, 3, 4, and 5 years after treatment, respectively (Table 3, Supplementary Table 1).

On multivariate analysis of patients without HCC recurrence within 1 year of treatment, combined liver cirrhosis (HR, 2.404; 95\% CI, 1.912 to 3.024; $\mathrm{p}<0.001$ ), treated with RFA rather than resection (HR, 1.705; 95\% CI, 1.395 to 2.084; $\mathrm{p}<0.001$ ), multiple tumors (HR, 1.328; 95\% CI, 1.050 to $1.678 ; \mathrm{p}=0.018$ ), and higher MELD score (HR, 1.068; 95\% CI, 1.025 to 1.113; $\mathrm{p}=0.002$ ) at time of treatment were significantly associated with increased risk of HCC recurrence from 1 year after treatment. Multivariate analysis of predictors of HCC recurrence from 2 years after treatment showed similar results with those of HCC recurrence from 1 year after treatment (Table 3).

In patients without $\mathrm{HCC}$ recurrence within 3 years of treatment, older age (HR, 1.020; 95\% CI, 1.002 to 1.038 ; $\mathrm{p}=0.026$ ), combined liver cirrhosis (HR, 3.136; 95\% CI, 2.066 to $4.759 ; \mathrm{p}<0.001)$, and treated with RFA rather than resection (HR, 1.872; 95\% CI, 1.290 to $2.717 ; \mathrm{p}=0.001$ ) were significantly associated with an increased risk of HCC recurrence from 3 years after treatment. Combined liver cirrhosis (HR, 2.846; 95\% CI, 1.692 to 4.787; $\mathrm{p}<0.001$ ) and treated with RFA rather than resection (HR, 1.651; 95\% CI, 1.011 to $2.697 ; \mathrm{p}=0.045)$ were independent predictors for HCC recurrence from 4 years. Older age (HR, 1.042; 95\% CI, 1.005 to $1.079 ; \mathrm{p}=0.024$ ) and combined liver cirrhosis (HR, 3.167; 95\% CI, 1.445 to $6.891 ; \mathrm{p}=0.004$ ) were independent predictors for HCC recurrence from 5 years. 


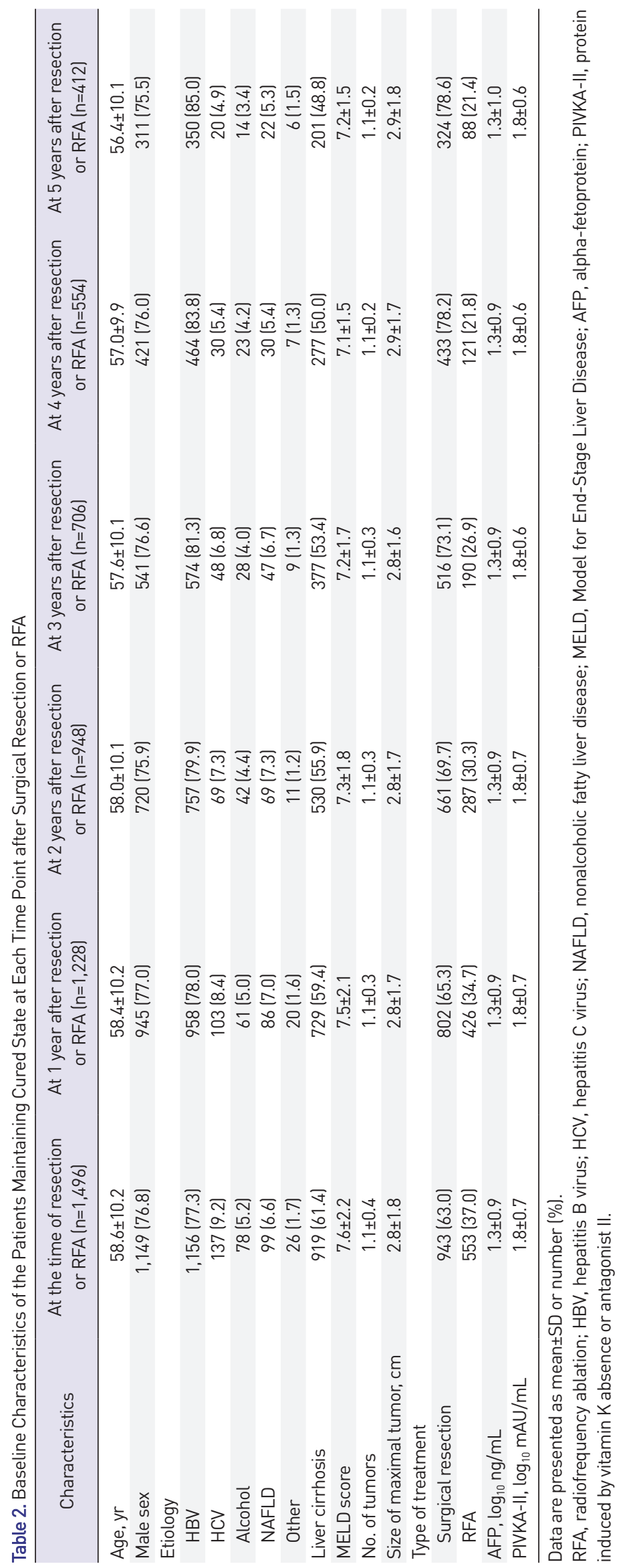




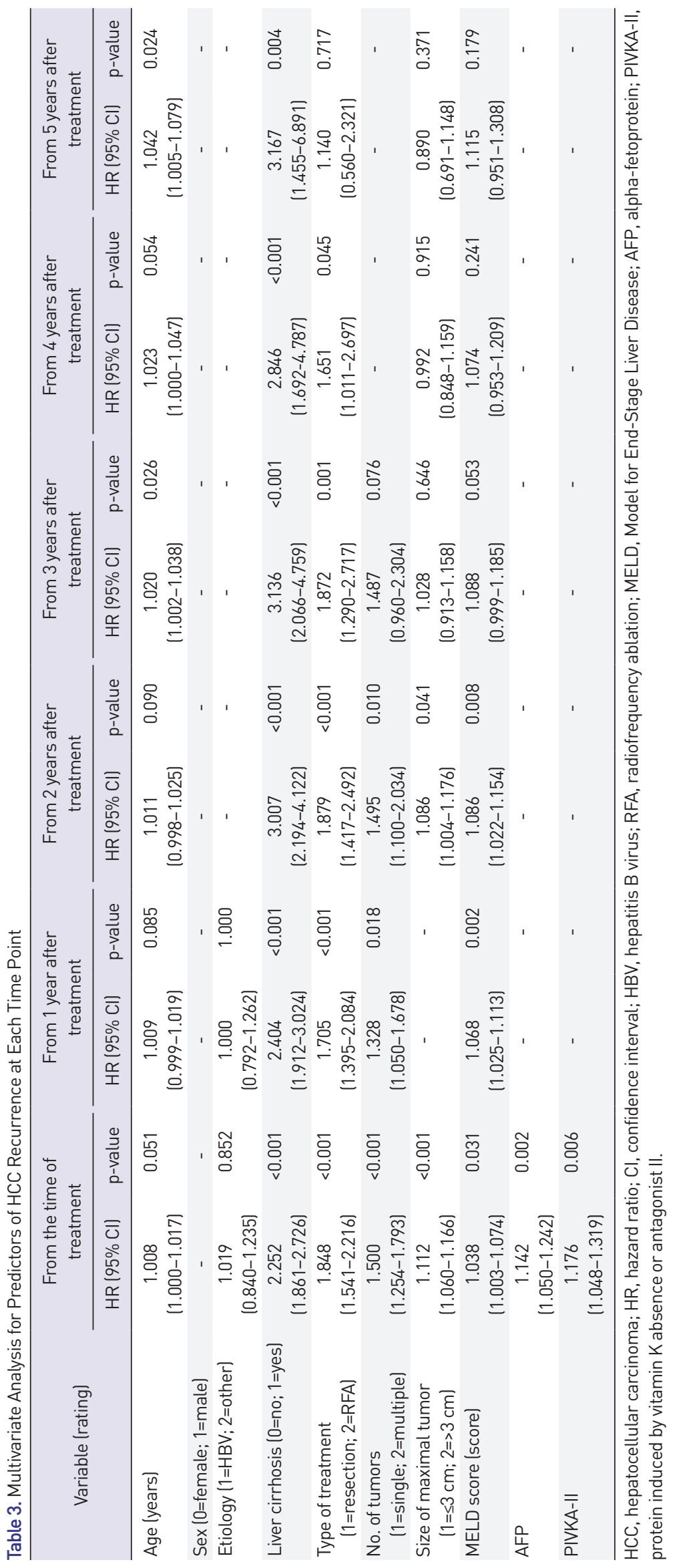




\section{DISCUSSION}

The changes of pattern and independent predictors of HCC recurrence after curative treatment with time progression arouse the confusion in differentiation of early and late recurrence and thereby, the establishment of surveillance strategy for HCC recurrence. In our large multicenter retrospective cohort study, we enrolled patients with first-time diagnosis of BCLC A HCC and who were completely cured with surgical resection or RFA. The cumulative incidence rates of HCC recurrence decreased gradually as time progressed. Liver cirrhosis and treated with RFA rather than resection were independently associated with increased risk of HCC recurrence from almost all time points, whereas multiple tumors, maximal tumor size $\geq 3 \mathrm{~cm}$, and higher MELD score were independently associated with increased risk of HCC recurrence only from within 2 years after curative treatment.

Our study comes with several unique strengths and clinical implications. First, we included the largest number $(n=1,491)$ of patients to our knowledge. Previous studies evaluating the change of HCC recurrence pattern have assessed a maximum of 816 patients. ${ }^{12,14,15,17}$ Besides, in contrast to other studies that only included patients treated with surgical resection, ${ }^{12,14,15,17,28}$ we recruited patients treated with both surgical resection and RFA. The diversity and large size of this study population enabled us to effectively compare the changes in recurrence patterns and independent predictors of HCC recurrence.

Further, the cumulative incidence rates of HCC recurrence gradually decreased with time. This result suggests that the surveillance interval could be gradually prolonged as time progression. However, we found significant differences in incidence rates of HCC recurrence between patients with and without cirrhosis, and between patients treated with surgical resection and RFA (all $\mathrm{p}<0.001$ ). These differences indicate that the interval periods should be adjusted to each patient depending on the cirrhosis and type of treatment.

This study clearly showed that tumor-related factors were independently associated with increased risk of HCC recurrence from only within 2 years of treatment. Multiple tumors and maximal tumor size $\geq 3 \mathrm{~cm}$ were independently associated with the increased risk of HCC recurrence from the time within 2 years after treatment. Serum AFP and PIVKA-II levels were independently associated with the increased risk of HCC only from the time of treatment. Bearing in mind the different pathogenesis of early and late recurrence, it is conceivable that tumor factors do not affect the development of late recurrence.

The difference in predictors for early and late recur- rence have been evaluated in several previous studies. However, classical concept of liver-related and tumorrelated factors have not been clearly revealed than we have expected. Imamura et al. ${ }^{12}$ showed that multiple tumors and gross tumor classification were significantly associated with late recurrence in patients treated with surgical resection. Wu et al. ${ }^{14}$ also suggested that multinodularity of tumor was significant predictor for both early and late recurrence in patients with HBV-related HCC. This discrepancy could be explained by the exclusion of presence of liver cirrhosis from the analysis variables. Also, it was surprising that patients without cirrhosis have shown to have similar prognosis with that of patients with cirrhosis. ${ }^{14}$ Cheng et al. ${ }^{17}$ indicated that AFP $>400 \mathrm{ug} / \mathrm{L}$ was independently associated with late recurrence in patients with solitary HCC and treated with partial hepatectomy, suggesting that these results were possibly due to the association of cirrhosis and elevated serum AFP level. However, this suggestion does not seem to be persuasive, because liver cirrhosis was the other independent predictor of late recurrence. Though many previous studies ${ }^{12,14,17}$ indicated the tumorrelated factors as significant predictors for late recurrence, it seems that these disparities have been arisen from the heterogeneity of HCC stage in enrolled patients. The classical concept for liver and tumor-related factors could be ideally elucidated in early stage HCC, because advanced stage HCC may not be completely removed after successful curative treatment, and microscopic intrahepatic metastases often remain. Therefore, we only included patients with BCLC stage A HCC to clearly prove this classical concept.

For all the time points analyzed after treatment, liver cirrhosis emerged as a potent independent predictor of HCC recurrence (all $\mathrm{p}<0.05)$. These results were consistent with the previously mentioned studies. ${ }^{1728}$ In fact, late recurrence is specifically considered to be due to the development of second primary HCC after treatment at primary sites, and this field effect plays a critical role in hepatocarcinogenesis. ${ }^{29}$ Therefore, it can be concluded that in patients with liver cirrhosis, the risk of second primary HCC development remains high even after successive curative treatment.

We also found that treatment with RFA was an independent predictor of HCC recurrence form almost all time points after treatment. Because the type of treatment is decided based on both tumor and liver disease related factors, it could reflect both tumor- and liver disease-related factors. Patients advised to undergo RFA tend to have more advanced stages of liver dysfunction, older age and have more comorbidities than those who are advised to undergo surgical resection. These features might cause increased incidence of both early and late recurrence in patients treated 
with RFA rather than in patients treated with resection.

Finally, when considering the time point when the tumor-related factors lose their prognostic implication for HCC recurrence, 2 years after treatment may be used as the period defining early and late recurrence of HCC, consistent with the classical belief. ${ }^{10,11}$ Therefore, it is acceptable that the duration with sustained clinical implication of tumor-related factors could be the optimal cutoff point for differentiating early and late recurrence of HCC. Thus, this study has made an important clinical contribution towards defining the period of early recurrence as 2 years after treatment, because there has been no sufficient evidence yet.

There are several limitations and issues that remain unaddressed. First, the retrospective design of our study allows for potential selection bias and lead-time bias. Second, a large proportion of patients treated with RFA suffered from early recurrence or death, leaving only 88 patients treated with RFA for analysis at 5 years after treatment. Therefore, the prognostic significance of RFA would not be observed in recurrence from 5 years after treatment. Third, the MELD score, which represents the degree of liver dysfunction, was significantly associated only with early recurrence in this study. This disparity could be explained by the earlier death of patients with higher MELD score. Lastly, majority of patients had HBV infection (77.3\%). It has been well known that $62.2 \%$ of HCC patients in Korea have HBV infection, ${ }^{30}$ and this could be important limitation of this study. Further studies in different population would be needed for general application of this result.

In conclusion, we demonstrated a gradual decrease in the incidence rates of HCC recurrence after curative treatment in patients with BCLC A HCC. Tumor-related factors were independently associated with increased risk of HCC recurrence from within 2 years after treatment. Therefore, the period of 2 years after curative treatment may be used to differentiate early and late recurrence of HCC.

\section{CONFLICTS OF INTEREST}

S.U.K. is an editorial board member of the journal but did not involve in the peer reviewer selection, evaluation, or decision process of this article. No other potential conflicts of interest relevant to this article were reported.

\section{AUTHOR CONTRIBUTIONS}

Conception and design: H.A.L., S.U.K., Y.S.S. Development of methodology: H.A.L., S.U.K., Y.S.S. Acquisition, analysis and interpretation of data: H.A.L., S.U.K., Y.S.S. Writing, review, and/or revision of the manuscript: H.A.L., Y.S.L., B.K.K., Y.K.J., J.Y.P., J.H.K., H.A., D.Y.K., H.J.Y., S.H.A., J.E.Y., K.S.B., K.H.H., S.H.U., S.U.K., Y.S.S. Administrative, technical, or material support: S.U.K., Y.S.S. Study supervision: S.U.K., Y.S.S.

\section{ORCID}

Han Ah Lee https://orcid.org/0000-0002-0430-1607 Young-Sun Lee https://orcid.org/0000-0001-6396-0859 Beom Kyung Kim https://orcid.org/0000-0002-5363-2496 Young Kul Jung https://orcid.org/0000-0002-6566-1382 Seung Up Kim https://orcid.org/0000-0002-9658-8050 Jun Yong Park https://orcid.org/0000-0003-1990-4643 Ji Hoon Kim https://orcid.org/0000-0003-3924-0434 Hyunggin An https://orcid.org/0000-0002-0566-758X Do Young Kim https://orcid.org/0000-0003-2395-0183 Hyung Joon Yim https://orcid.org/0000-0002-6036-2754 Sang Hoon Ahn https://orcid.org/0000-0002-3629-4624 Jong Eun Yeon https://orcid.org/0000-0002-0510-7371 Kwan Soo Byun https://orcid.org/0000-0003-4031-842X Kwang-Hyub Han https://orcid.org/0000-0003-3960-6539 Soon Ho Um https://orcid.org/0000-0002-1369-0974 Yeon Seok Seo https://orcid.org/0000-0003-4171-6331

\section{REFERENCES}

1. Global Burden of Disease Liver Cancer Collaboration, Akinyemiju T, Abera $S$, et al. The burden of primary liver cancer and underlying etiologies from 1990 to 2015 at the global, regional, and national level: results from the Global Burden of Disease Study 2015. JAMA Oncol 2017;3:16831691.

2. Korean Liver Cancer Association; National Cancer Center. 2018 Korean Liver Cancer Association-National Cancer Center Korea practice guidelines for the management of hepatocellular carcinoma. Gut Liver 2019;13:227-299.

3. Colombo M. EASL clinical practice guidelines for the management of occupational liver diseases. Liver Int 2020;40 Suppl 1:136-141.

4. Heimbach JK, Kulik LM, Finn RS, et al. AASLD guidelines for the treatment of hepatocellular carcinoma. Hepatology 2018;67:358-380.

5. Arii S, Tanaka J, Yamazoe Y, et al. Predictive factors for intrahepatic recurrence of hepatocellular carcinoma after partial hepatectomy. Cancer 1992;69:913-919.

6. Izumi R, Shimizu K, Ii T, et al. Prognostic factors of hepatocellular carcinoma in patients undergoing hepatic resection. 
Gastroenterology 1994;106:720-727.

7. Koike Y, Shiratori Y, Sato S, et al. Risk factors for recurring hepatocellular carcinoma differ according to infected hepatitis virus-an analysis of 236 consecutive patients with a single lesion. Hepatology 2000;32:1216-1223.

8. Lise M, Bacchetti S, Da Pian P, Nitti D, Pilati PL, Pigato P. Prognostic factors affecting long term outcome after liver resection for hepatocellular carcinoma: results in a series of 100 Italian patients. Cancer 1998;82:1028-1036.

9. Poon RT, Fan ST, Lo CM, Liu CL, Wong J. Long-term survival and pattern of recurrence after resection of small hepatocellular carcinoma in patients with preserved liver function: implications for a strategy of salvage transplantation. Ann Surg 2002;235:373-382.

10. Sherman M. Recurrence of hepatocellular carcinoma. N Engl J Med 2008;359:2045-2047.

11. Llovet JM, Schwartz M, Mazzaferro V. Resection and liver transplantation for hepatocellular carcinoma. Semin Liver Dis 2005;25:181-200.

12. Imamura $\mathrm{H}$, Matsuyama $\mathrm{Y}$, Tanaka E, et al. Risk factors contributing to early and late phase intrahepatic recurrence of hepatocellular carcinoma after hepatectomy. J Hepatol 2003;38:200-207.

13. Hoshida Y. Risk of recurrence in hepatitis B-related hepatocellular carcinoma: impact of viral load in late recurrence. J Hepatol 2009;51:842-844.

14. Wu JC, Huang YH, Chau GY, et al. Risk factors for early and late recurrence in hepatitis B-related hepatocellular carcinoma. J Hepatol 2009;51:890-897.

15. Hirokawa F, Hayashi M, Asakuma M, Shimizu T, Inoue Y, Uchiyama K. Risk factors and patterns of early recurrence after curative hepatectomy for hepatocellular carcinoma. Surg Oncol 2016;25:24-29.

16. Utsunomiya T, Shimada M, Imura S, Morine Y, Ikemoto T, Mori M. Molecular signatures of noncancerous liver tissue can predict the risk for late recurrence of hepatocellular carcinoma. J Gastroenterol 2010;45:146-152.

17. Cheng Z, Yang P, Qu S, et al. Risk factors and management for early and late intrahepatic recurrence of solitary hepatocellular carcinoma after curative resection. HPB (Oxford) 2015;17:422-427.
18. Hoshida Y, Villanueva A, Kobayashi M, et al. Gene expression in fixed tissues and outcome in hepatocellular carcinoma. N Engl J Med 2008;359:1995-2004.

19. Hanouneh IA, Alkhouri N, Singal AG. Hepatocellular carcinoma surveillance in the 21st century: saving lives or causing harm? Clin Mol Hepatol 2019;25:264-269.

20. Lok AS, McMahon BJ. Chronic hepatitis B. Hepatology 2007;45:507-539.

21. European Association for the Study of the Liver. EASL clinical practice guidelines: management of chronic hepatitis $B$ virus infection. J Hepatol 2012;57:167-185.

22. European Association for the Study of the Liver. EASL clinical practice guidelines: management of chronic hepatitis B. J Hepatol 2009;50:227-242.

23. Korean Association for the Study of the Liver. KASL clinical practice guidelines: management of chronic hepatitis B. Clin Mol Hepatol 2012;18:109-162.

24. Kim TH, Kim SY, Tang A, Lee JM. Comparison of international guidelines for noninvasive diagnosis of hepatocellular carcinoma: 2018 update. Clin Mol Hepatol 2019;25:245-263.

25. Forner A, Reig M, Bruix J. Hepatocellular carcinoma. Lancet 2018;391:1301-1314.

26. Ahn KS, Kang KJ. Appropriate treatment modality for solitary small hepatocellular carcinoma: radiofrequency ablation vs. resection vs. transplantation? Clin Mol Hepatol 2019;25:354-359.

27. Lencioni R, Llovet JM. Modified RECIST (mRECIST) assessment for hepatocellular carcinoma. Semin Liver Dis 2010;30:52-60.

28. Yamamoto Y, Ikoma H, Morimura R, et al. Optimal duration of the early and late recurrence of hepatocellular carcinoma after hepatectomy. World J Gastroenterol 2015;21:12071215.

29. Suk KT, Baik SK, Yoon JH, et al. Revision and update on clinical practice guideline for liver cirrhosis. Korean J Hepatol 2012;18:1-21.

30. Kim BH, Lim YS, Kim EY, et al. Temporal improvement in survival of patients with hepatocellular carcinoma in a hepatitis B virus-endemic population. J Gastroenterol Hepatol 2018;33:475-483. 